\title{
Scotopic Electroretinogram in Term Infants Born of Mothers Supplemented with Docosahexaenoic Acid during Pregnancy
}

\author{
Cari A. Malcolm, ${ }^{1}$ Ruth Hamilton, ${ }^{2}$ Daphne L. McCulloch, ${ }^{1}$ Colette Montgomery, ${ }^{3}$ and \\ Lawrence T. Weaver ${ }^{3}$
}

Purpose. To test the hypothesis that the supplementation of the diets of pregnant women with a fish oil rich in docosahexaenoic acid (DHA) enhances retinal development in their healthy term infants, as measured during the early postnatal period by the electroretinogram (ERG).

METHODs. One hundred pregnant women were randomized to receive either a fish oil $(n=50)$ or a placebo oleic acid dietary supplement $(n=50)$ from 15 weeks of pregnancy until delivery. Total fatty acids in red blood cells (RBCs) and plasma were measured in mothers at 15 and 28 weeks of pregnancy and at delivery and in their infants in umbilical cord blood. Infant retinal development was assessed within the first week of life with full-field ERGs that included a scotopic blue intensity series $(n=41)$ and a bright white flash $\left(2.0 \log \mathrm{cd}-\mathrm{s} / \mathrm{m}^{2} ; n=\right.$ 44).

RESults. Infants born of mothers who received supplements did not differ at birth in weight, gestational age, or any other standard variable. Infant DHA status at birth, as measured from umbilical cord blood, did not differ significantly between maternal supplementation groups. ERG implicit times, amplitudes, and parameters of the stimulus-response function did not differ significantly between infants in the maternal supplemented and placebo groups. There was, however, a relationship between infant DHA status and maturity of the retina at birth, regardless of maternal supplementation group. A measure of retinal sensitivity $(\log \sigma)$ correlated significantly $(P<$ 0.005 ) with DHA status (as a percentage of total fatty acid; TFA) in infant cord blood. Infants in the highest quartile for cord blood DHA had higher retinal sensitivity compared with infants in the lowest quartile. Infants in the highest quartile for plasma DHA, both as a percentage of TFA and concentration, were born at a significantly later gestational age than were infants in the lower quartiles.

Conclusions. These findings demonstrate an association between the DHA status of term infants and retinal sensitivity, suggesting an essential role of this long-chain polyunsaturated fatty acid (LCPUFA) in the development and function of the

From the ${ }^{1}$ Department of Vision Sciences, Glasgow Caledonian University, Glasgow, United Kingdom; and the Departments of ${ }^{2} \mathrm{Clini}-$ cal Physics and ${ }^{3}$ Child Health, University of Glasgow, Royal Hospital for Sick Children, Glasgow, United Kingdom.

Supported by Grant K/MRS/50/C2730 from the Chief Scientist's Office, Scottish Office Health Department.

Submitted for publication July 30, 2002; revised January 29, 2003; accepted February 28, 2003.

Disclosure: C.A. Malcolm, None; R. Hamilton, None; D.L. McCulloch, None; C. Montgomery, None; L.T. Weaver, None

The publication costs of this article were defrayed in part by page charge payment. This article must therefore be marked "advertisement" in accordance with 18 U.S.C. $\$ 1734$ solely to indicate this fact.

Corresponding author: Cari A. Malcolm, Department of Child Health, University of Glasgow, Yorkhill, Glasgow G3 8SJ, UK; gcl305@clinmed.gla.ac.uk. retina. However, maternal DHA status was not significantly associated with infant retinal sensitivity and no direct effect of maternal supplementation was observed. (Invest Ophthalmol Vis Sci. 2003;44:3685-3691) DOI:10.1167/iovs.02-0767

$\mathrm{T}$ he highest concentration of docosahexaenoic acid (DHA) in the body is found in the outer segment membranes of the photoreceptors, ${ }^{1,2}$ suggesting that this long-chain polyunsaturated fatty acid (LCPUFA) plays essential structural and functional roles within the human retina. During the last few months of fetal life and the early months of postnatal life, large quantities of DHA are deposited in the infant brain and retina. ${ }^{3,4}$ Because the photoreceptor outer segment membranes are in a state of rapid growth during this period, the developing retina has a particularly high DHA requirement and may be vulnerable to deficiencies. With a limited ability to synthesize this LCPUFA from its precursors, the infant relies on maternal sources of DHA, prenatally through active transport across the placenta and postnatally in breast milk.

Over the past few decades, adverse effects of reduced DHA concentration on retinal function have been demonstrated in studies conducted in both animals ${ }^{5-10}$ and humans. ${ }^{11,12}$ Most of these studies have used the ERG to assess the effects of postnatal DHA deficiency and/or supplementation on retinal function. In a study of very-low-birth-weight (VLBW) and premature infants tested at 36 weeks of postconceptional age (PCA), those infants receiving formulas without DHA demonstrated significantly lower rod maximum amplitudes, lower sensitivity, and higher thresholds than those infants receiving DHA-supplemented formulas. ${ }^{11}$ Such findings suggest that the maturity and sensitivity of the rod photoreceptors is delayed in premature infants who receive diets low in DHA. ${ }^{11}$

Before the present study, the potential effects of supplementing maternal diets with DHA during pregnancy on the development and maturation of the retina had not been examined. Increasing maternal DHA supply during pregnancy through dietary supplementation may increase the amount of DHA available to the developing infant. ${ }^{13,14}$ Subsequently, the increased DHA level in the infant may contribute to improved retinal and visual function. Therefore, the primary purpose of our study was to test the hypothesis that the dietary supplementation of pregnant women with fish oil rich in DHA enhances retinal function of their infants as measured by the ERG. The effect of maternal supplementation on maternal and infant DHA status, as reflected in maternal RBC and plasma and umbilical cord RBCs and plasma, is summarized herein and will be reported fully elsewhere. ${ }^{15,16}$

\section{MeTHODS}

\section{Experimental Design and Subjects}

This study was prospective, double-blind, randomized, and controlled in design. It was performed in accordance with the Declaration of Helsinki and approved by the Ethics Committee of Yorkhill NHS Trust 
Table 1. The Fatty Acid Composition of the Fish Oil and the High Oleic Sunflower Oil Placebo Study Supplements*

\begin{tabular}{lcc}
\hline Fatty Acid & $\begin{array}{c}\text { Fish Oil } \\
\text { (Marinol D40) }\end{array}$ & $\begin{array}{c}\text { High Oleic } \\
\text { Sunflower Oil }\end{array}$ \\
\hline C14:0 & 3.7 & - \\
C16:0 & 6.7 & 4.0 \\
C16:1n-7 & 4.3 & - \\
C18:0 & 2.4 & 5.0 \\
C18:1n-9 & 15.6 & 81 \\
C18:2n-6 & 1.2 & 7.2 \\
C18:3n-3 & 0.8 & - \\
C18:4 & 1.4 & - \\
C20:0 & 0.1 & - \\
C20:1 & 2.0 & - \\
C20:5n-3 & 7.2 & - \\
C22:1 & 2.7 & - \\
C22:5n-6 & 4.1 & 97.2 \\
C22:6n-3 & 40.4 & 3.0 \\
Sum of all & 92.6 & \\
Others & 9.4 & \\
\hline
\end{tabular}

Data are percent of total fatty acids.

* Marinol D40.

(Glasgow, UK). One hundred healthy pregnant women were invited to take part in the study while attending an antenatal clinic at approximately 15 weeks of pregnancy. Women with diabetes, twin pregnancies, pre-eclampsic toxemia, a history of abruption or postpartum hemorrhage, allergy to fish products, or a thrombophilic tendency or those receiving drugs that affect thrombocyte function (nonsteroidal anti-inflammatory drugs) were excluded. Written informed consent was obtained from each study participant at the time of enrollment.

The pregnant women were randomized to either the treatment or control group. Subjects in the treatment group $(n=50)$ received dietary supplementation with fish oil capsules rich in DHA (Marinol D40; $100 \mathrm{mg}$ of DHA per capsule; R. P. Scherer Ltd., Swindon, UK), whereas subjects in the control group $(n=50)$ received sunflower oil placebo capsules from enrollment until the time of delivery. The placebo capsules contained no traces of DHA or its precursor $\alpha$-linolenic acid (ALA). The fatty acid composition of the study capsules is described in Table 1. The fish oil and placebo capsules were identical in appearance and could not be distinguished by odor or taste. Subjects in both groups were instructed to consume two study capsules per day. Maternal diet, particularly the consumption of fish products, was assessed by interview at 15 and 28 weeks of pregnancy and at delivery.

Healthy infants born after 36 weeks' gestation, with an Apgar score of greater than seven at 5 minutes and without any diagnosed visual, medical, or developmental disorders were eligible to continue in the postnatal phase of the study. Infant retinal function was assessed as soon as possible after birth, but always within the first week of life, using full-field electroretinograms (ERGs). Birth weight, length, and occipital frontal head circumference (OFC) were measured within the first 24 hours after delivery.

\section{Fatty Acid Composition}

Venous blood samples were obtained from the women at enrollment (15 weeks of pregnancy), at 28 weeks of pregnancy, and at delivery. Blood samples were also obtained from the umbilical cord at delivery. Total fatty acids in plasma and red blood cells (RBCs) were extracted by modified Folch extraction and derivatized with methanolic hydrochloric acid. Fatty acid methyl esters were analyzed by gas chromatography mass spectrometry (GC-MS). The results were expressed in both relative (percentage of total fatty acids, \%TFA) and absolute (concentrations in nanomoles per milliliter) terms.

\section{Electroretinography}

ERG recordings were coordinated with the infants' feeding patterns, ideally after a feeding, when infants were most likely to be settled or asleep. Before dark adaptation, the pupils were dilated with 1 drop of $0.5 \%$ cyclopentolate in each eye. Sterile, light-occluding adhesive patches were applied, and the infant was allowed to dark adapt for a minimum of 30 minutes. At the end of the dark adaptation period, the infant was transferred to the recording room. All ERG recordings took place in a side room of the neonatal unit in the Queen Mother's Hospital, Yorkhill NHS Trust (Glasgow, UK). The infant was swaddled and placed supine on a specially made foam mattress inside an incubator. The incubator had been modified with a customized aperture in the cover to allow the Ganzfeld bowl (Ganzfeld Stimulator; LKC Systems Inc., Gaithersburg, MD) to be lowered and positioned directly over the infant's face. The distance from the viewing aperture of the Ganzfeld bowl to the infant's eyes was 8 to $10 \mathrm{~cm}$, and the diameter of the Ganzfeld bowl opening was $27 \mathrm{~cm}$.

The infant's forehead was cleansed with a skin preparation gel $(\mathrm{Nu}$ Prep; D. O. Weaver and Co., Aurora, CO). Conductive paste (10-20; D. O. Weaver and Co.) was applied to a silver-silver chloride disc electrode, and the electrode was placed centrally on the forehead to act as a ground. Under dim red light, the adhesive eye patches were removed, and pupil dilatation with 1 drop of $0.5 \%$ cyclopentolate in each eye was repeated. One drop of $0.5 \%$ proxymetacaine, to act as a topical anesthetic, was instilled in the right eye. The recording electrode, a bipolar Burian-Allen contact lens electrode, sized especially for a small infant (Hansen Ophthalmic Department Laboratories, Iowa City, IA), was filled with $1 \%$ carmellose sodium (Celluvisc; Allergan Pharmaceuticals Ltd., Westport, Ireland) to act as a lubricant and placed on the right cornea.

Stimuli were delivered into the Ganzfeld bowl by a photostimulator (Grass PS33 Plus; Astro-Med Inc., West Warwick, RI) set at its maximum intensity. The signals were amplified and filtered by an amplifier (Biodata PA400; Biodata, Manchester, UK) with a band-pass of 0.5 to $300 \mathrm{~Hz}$ and a gain of 5000. The amplified signals were then processed in real time (averaging and artifact rejection) with custom-designed software. ${ }^{17}$

An intensity-series ERG was performed to study rod photoreceptor function. A series of calibrated neutral-density filters (Wratten; Eastman Kodak, Herts, UK) mounted on a filter wheel and a blue filter were used to record rod ERGs to progressively increasing flash brightness. Ganzfeld luminance ranged from $-4.475 \mathrm{log} \mathrm{scot} \mathrm{cd}-\mathrm{s} / \mathrm{m}^{2}$ to $0.442 \mathrm{log}$ scot cd-s $/ \mathrm{m}^{2}$ (peak wavelength, $475 \mathrm{~nm}$ ) in 0.4-log unit steps. At higher intensities, cone intrusion into the rod response can occur. For this reason, the procedure was repeated using a photopically matched red filter (peak wavelength, $650 \mathrm{~nm}$ ), evoking mixed rod and cone ERGs, and these responses were computer-subtracted from the responses to blue flashes to achieve rod-only responses at higher troland levels. ${ }^{18}$ The photopic match was established by photometric calibration of the filters and by matching of adult photopic ERG amplitudes to red and blue stimuli.

In addition, an ERG was recorded under dark-adapted conditions to a bright white flash $\left(2.0 \mathrm{log} \mathrm{cd}-\mathrm{s} / \mathrm{m}^{2}\right)$ to elicit a mixed rod and cone

TABLE 2. Confirmation of Equivalence of a Neonatal and an Adult Troland by Comparison of PD and PND Ratios between Age Groups

\begin{tabular}{lcc}
\hline & $\begin{array}{c}\text { Mean Dilated } \\
\text { Pupil Diameter } \\
(\mathbf{m m})\end{array}$ & $\begin{array}{c}\text { Posterior } \\
\text { Nodal Distance } \\
(\mathbf{m m})\end{array}$ \\
\hline Newborns & 5.0 & $11.0^{*}$ \\
Adults & 8.0 & 16.7 \\
Ratio & 0.63 & 0.66 \\
2-Month infants & $5.2 \dagger$ & $11.2 \dagger$ \\
Adults & $7.2 \dagger$ & $16.7 \dagger$ \\
Ratio & $0.72 \dagger$ & $0.67 \dagger$ \\
\hline
\end{tabular}

${ }^{*}$ Data from Isenberg et al., $1995 .{ }^{22}$

† Data from Brown et al., 1987. ${ }^{21}$ Pupils in this study were not dilated, but were measured while fully dark adapted. 


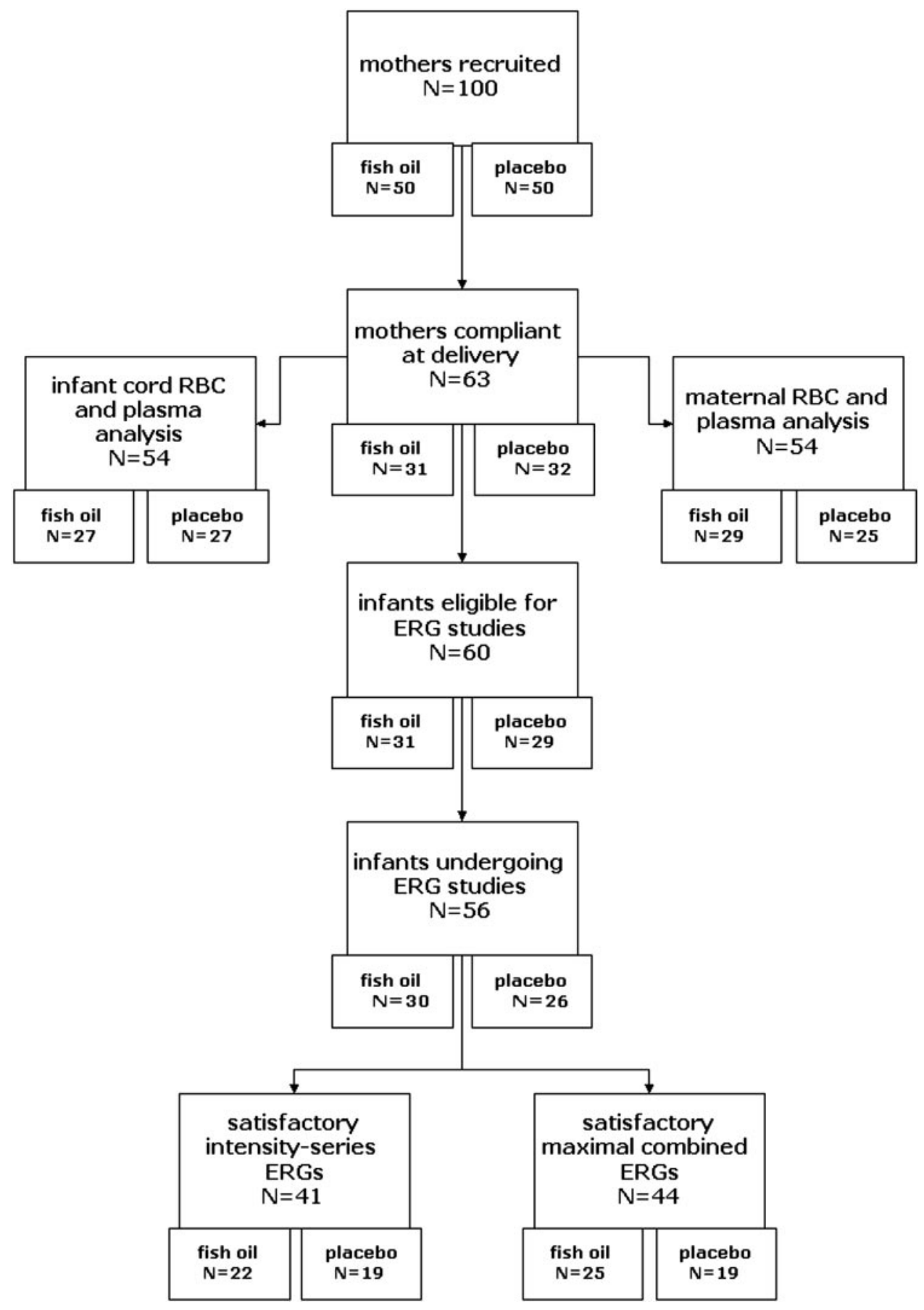

Figure 1. Flowchart showing the number of mothers and infants remaining at each stage of investigation.

signal, which matches the International Society for Clinical Electrophysiology of Vision (ISCEV) standard maximum combined ERG. ${ }^{19}$

All calibrations were performed with an integrating radiometer with either photopic or scotopic photometric filters (IL1700 Radiometer; International Light Inc., Newburyport, MA).

The interstimulus interval was 5 seconds for dim flashes and 10 seconds for bright flashes, to allow full recovery of the b-wave amplitude between flashes. Two to 30 ERGs were averaged at each intensity. The infants were monitored with a dim red light, and the flashes were triggered when the pupil was clearly visible within the contact lens electrode. At the end of testing, pupil diameter was measured and recorded in millimeters. The complete recording procedure, excluding dark adaptation, lasted approximately 45 to 60 minutes.
Recordings were performed in 11 adults (ages, 26-41 years) with an identical protocol, but with the adults sitting upright looking into the Ganzfeld bowl and wearing an adult-sized Burian-Allen electrode.

The ERG waveform has two main components, a negative a-wave followed by a positive b-wave. Amplitudes and implicit times of these components were measured for each ERG according to methods described in the ISCEV standards. ${ }^{19}$ The a-wave amplitude was measured from the baseline to the a-wave trough, whereas the b-wave amplitude was measured from the a-wave trough to the b-wave peak. Implicit time of both the a- and b-waves was measured from the time of the flash stimulus to the peak or trough of the response.

Rod photoreceptor function was examined by fitting the b-wave amplitudes to the Naka-Rushton function ${ }^{20}: V / V_{\max }=I^{n} /\left(I^{n}+\sigma^{n}\right)$, 
TABLe 3. Characteristics of Infants in the Maternal Fish Oil and Placebo Groups Eligible for ERG Studies

\begin{tabular}{lccc}
\hline \multicolumn{1}{c}{ Characteristic } & $\begin{array}{c}\text { Fish Oil } \\
(\boldsymbol{n}=\mathbf{3 1})\end{array}$ & $\begin{array}{c}\text { Placebo } \\
(\boldsymbol{n}=29)\end{array}$ & $\boldsymbol{P}$ \\
\hline Gestational age at birth (days) & $279.7 \pm 9.5$ & $279.6 \pm 8.5$ & 0.96 \\
Gender (male/female) & $16 / 15$ & $11 / 18$ & 0.21 \\
Birth weight (g) & $3508 \pm 501$ & $3645 \pm 495$ & 0.29 \\
Birth length (cm) & $52.6 \pm 2.5$ & $53.2 \pm 2.9$ & 0.42 \\
Birth OFC (cm) & $34.8 \pm 1.4$ & $34.9 \pm 1.5$ & 0.86 \\
\hline
\end{tabular}

Data are the mean $\pm \mathrm{SD}$.

where $V=$ b-wave amplitude expressed in microvolts, $I=$ retinal illuminance energy in scotopic troland-seconds (scot td-s) and $V_{\max }$, $\log \sigma$, and $n$ are the derived parameters. $V_{\max }$ is the maximum amplitude of the b-wave. $\log \sigma$, a measure of retinal sensitivity, is the flash energy at which $V_{\max }$ is half its maximum value. The exponent $n$ represents the slope of the curve and was constrained to equal 1 . To meet the criteria for analysis using the stimulus-response function, the ERG intensity series had to be reliably recorded to a minimum of five different light intensities. Goodness-of-fit was established using $r^{2}$ values.

Flash brightness incident on the retina (retinal illuminance energy), was expressed in scotopic troland-seconds (scot td-s) and calculated by multiplying the Ganzfeld luminance energy (in candela-seconds per square meter) by pupil diameter (in square millimeters). The equivalence of an adult and a neonatal troland was confirmed by comparison of the ratios of adult-to-infant pupil diameter (PD) and posterior nodal distances (PNDs) ${ }^{21,22}$ (Table 2), which were sufficiently similar (0.63 and 0.66 , respectively)

\section{Statistical Analysis}

Differences in intensity series ERG implicit times between groups were determined by two-factor repeated-measures ANOVA, with maternal supplementation group and retinal illuminance as the main factors. Differences in parameters of the stimulus-response function ( $V_{\max }$ and $\log \sigma$ ) between maternal supplementation groups were also determined by ANOVA. Linear regression analysis was performed to relate infant RBC and plasma LCPUFA status, specifically DHA, arachidonic acid (AA), total n-6 LCPUFAs, total n-3 LCPUFAs, and the n-6 to n-3 ratio, with the $b$-wave implicit times, parameters of the stimulusresponse function, and implicit times and amplitudes of the maximum combined ERG. To examine the data for nonlinear associations, a Kendall $\tau$ test was performed to test for a rank correlation between the $V_{\max }$ or $\log \sigma$ and LCPUFA quartiles, as measured from umbilical cord blood. Potential confounding variables, such as birth weight, gender, gestational age at birth and age (in hours) at time of testing were not significantly related to any measures of the ERGs evaluated in this study. Moreover, infants in the fish oil and placebo group did not differ significantly in birth weight, gender, gestational age at birth, and age at time of testing. Therefore, these variables were not included in the regression models.

All statistical analyses were performed on computer (SPSS for Windows, ver. 9.0; SPSS Inc., Chicago, IL).

\section{Results}

\section{Subjects}

One hundred women were recruited into the study at approximately 15 weeks of pregnancy (mean, 15.4 weeks or 109 days; range, 104-119). At study entry, subjects in the fish oil and placebo groups did not differ significantly in age, previous obstetric history, socioeconomic status, smoking habits, alcohol intake, exercise patterns or dietary intake of fish. Moreover, during the period between study entry and delivery, there were no significant differences between the groups in maternal dietary intake of fish or consumption of dietary supplements containing DHA $(P=0.31)$.

Figure 1 outlines the number of subjects remaining at each stage of the study. At delivery, 63 mothers and their infants remained in the study. One infant was withdrawn, because he was born at less than 36 weeks' gestation and two infants were withdrawn because they had low Apgar scores ( $<7$ at 5 minutes) at delivery and subsequently required admission to the special-care nursery for observation. Therefore, 60 infants, 31 in the fish oil and 29 in the placebo group were eligible to continue in the study. There were no significant differences in gestational length between the fish oil and placebo groups, with a mean gestational age of $279.7 \pm 9.5$ and $279.6 \pm 8.5$ days (SD), respectively. Moreover, birth weight, length, OFC, and the ratio of boys to girls were not significantly different between infants in the fish oil and placebo groups (Table 3 ). Gestational length, however, was significantly greater in infants in the upper quartile for umbilical cord plasma DHA, both as \%TFA $(P<0.005)$ and concentration $(P<0.05)$ when compared with infants in the lower quartiles (Table 4). Gestational length did not differ based on quartiles of umbilical cord RBC DHA.

ERGs were recorded in 56 (93\%) of the 60 study infants shortly after birth (mean $53.9 \pm 27.1$ hours). It was not possible to record ERGs from the remaining four infants because of early discharge of mothers and infants from the hospital $(n=$ $2)$, and withdrawal of consent for the ERG procedure $(n=2)$. Satisfactory ERG intensity series were obtained from 41 (73\%) of the 56 infants, 22 in the fish oil group and 19 in the placebo group. In the remaining 15 infants, fewer than five data points were obtained, and therefore they did not fulfill the criteria for analysis using the stimulus-response function. A satisfactory fit of the Naka-Rushton curves was evident with a median $r^{2}$ of 0.83 (range, 0.56-0 0.99). Maximum combined ERGs in response to a bright white flash were recorded in 44 (79\%) of the 56 infants, 25 in the fish oil group and 19 in the placebo group.

\section{Fatty Acid Composition in RBCs and Plasma}

Baseline DHA status, measured at enrollment, was similar between mothers in the fish oil and placebo supplementation groups. However, after receiving supplementation from approximately 15 weeks of pregnancy until delivery, the relative (\%TFA) and absolute (nanomoles per milliliter) amounts of DHA in RBCs was elevated in maternal RBCs in the fish oil group compared with the placebo group. At delivery, the median relative and absolute levels of DHA in mothers who received fish oil supplementation were $168.2 \mathrm{nmol} / \mathrm{mL}$ (range, $6.2-305.3$ ) and 3\% TFA (range, $0.3 \%-5.0$ ) compared with $118.9 \mathrm{nmol} / \mathrm{mL}$ (range, 17.3-225.8) and 2\% TFA (range, $0.5 \%-$ $4.0)$ in the mothers who received the placebo. There were no significant differences in umbilical cord blood DHA status between infants in the fish oil and placebo maternal supplementation groups. The median relative and absolute levels of DHA in infants born of the fish oil-supplemented mothers were 221

TABLE 4. Gestational Length in Days According to Umbilical Cord Plasma DHA Quartiles

\begin{tabular}{lll}
\hline $\begin{array}{c}\text { Umbilical Cord } \\
\text { Blood Quartiles }\end{array}$ & $\begin{array}{c}\text { Plasma DHA } \\
\text { Concentration } \\
\text { nmol/mL }\end{array}$ & $\begin{array}{c}\text { Plasma } \\
\text { DHA } \\
\text { (\% TFA) }\end{array}$ \\
\hline Quartile 1-low $(n=13)$ & $275.1 \pm 10.3$ & $273.8 \pm 7.9$ \\
Quartile 2-medium 1 $(n=14)$ & $279.9 \pm 3.9$ & $279.5 \pm 10.1$ \\
Quartile 3-medium 2 $(n=14)$ & $280.3 \pm 11.0$ & $282.1 \pm 7.6$ \\
Quartile 4-high $(n=13)$ & $283.5 \pm 6.1$ & $283.2 \pm 6.2$
\end{tabular}

Data are the mean $\pm \mathrm{SD}$ 

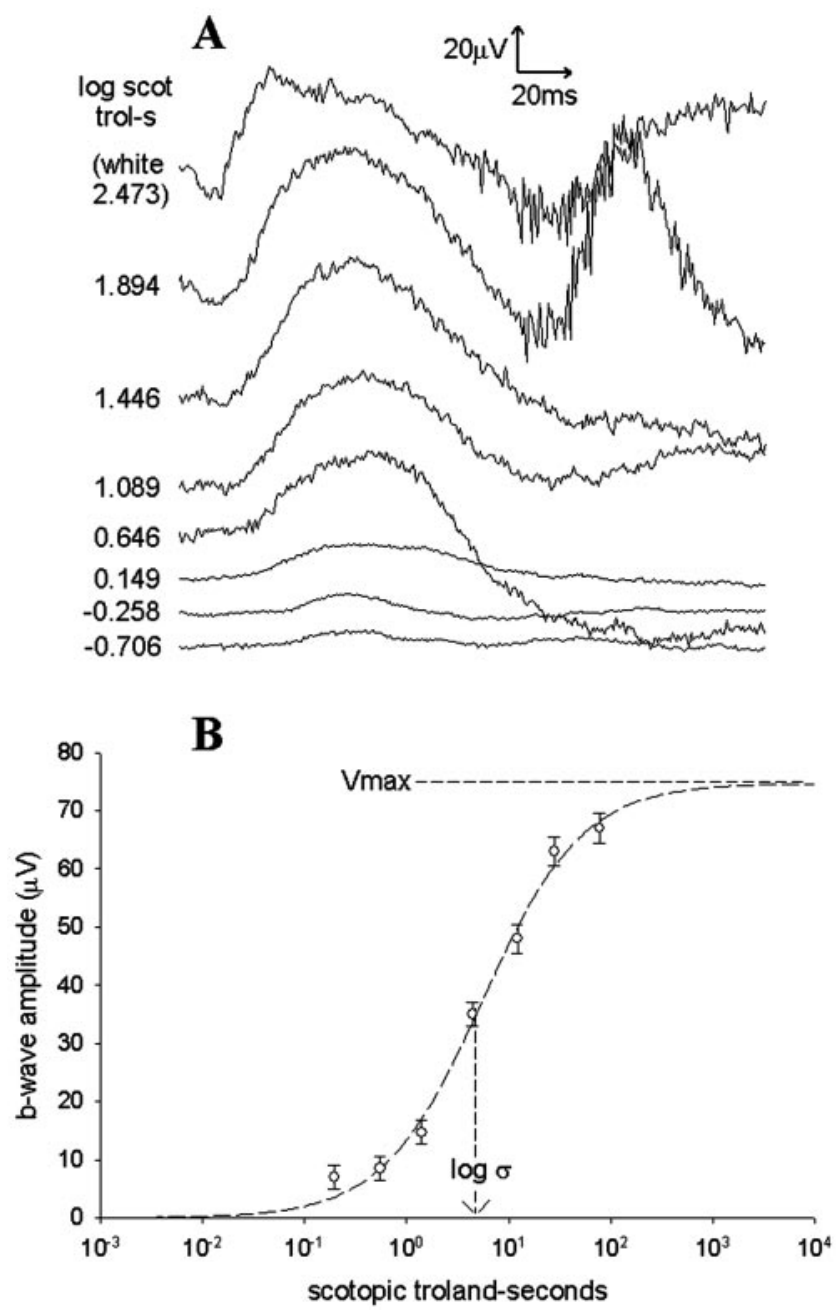

FigURE 2. Representative data: (A) scotopic intensity series recorded from a female infant born at 41.6 weeks' gestation and tested at 4 days of age. Troland level (log scotopic troland-seconds) is indicated in the small box to the right of the ERG signals. The top trace represents the maximum combined ERG, and was not used for the Naka-Rushton curve fitting. (B) The Naka-Rushton function, fit to the b-wave amplitudes $\left(V_{\max }\right)$ of the data.

nmol/mL (range, $70-465$ ) and 4.4\% TFA (range, 1.7\%-5.8\%) compared with $273 \mathrm{nmol} / \mathrm{mL}$ (range, 98-478) and 4.2\% TFA (range, $1.8 \%-6.2 \%$ ) in infants born of mothers who received the placebo.

\section{Electroretinography}

Intensity-Series ERG. Although a reproducible ERG intensity series can be successfully recorded in infants shortly after birth, according to procedures outlined in the international standards, ${ }^{19}$ our results demonstrate the relative immaturity of the dark-adapted ERG at term age. All infants had robust b-
TABLE 6. Quartiles for DHA in Umbilical Cord RBC for Those Infants Who Had an Intensity Series ERG Analyzed

\begin{tabular}{lcc}
\hline $\begin{array}{c}\text { Umbilical Cord } \\
\text { Blood Quartiles }\end{array}$ & $\begin{array}{c}\text { RBC Concentration } \\
\text { (nmol/mL) }\end{array}$ & $\begin{array}{c}\text { RBC } \\
\text { (\% TFA) }\end{array}$ \\
\hline Quartile 1-low & $141(70-180)$ & $3.4(1.7-3.7)$ \\
Quartile 2-medium 1 & $209(183-228)$ & $4.1(3.8-4.3)$ \\
Quartile 3-medium 2 & $286(240-321)$ & $4.5(4.4-4.8)$ \\
Quartile 4-high & $353(323-478)$ & $5.4(5.1-5.8)$ \\
\hline
\end{tabular}

Data are median (range).

waves for moderate or bright blue flashes. Cone intrusion was seldom evident, and red flashes elicited only small ERGs for the brighter flashes. The a-waves were small and once again evident only at the brighter flashes. A typical ERG intensity series recorded from a female term infant in response to a series of blue flashes and to the maximum white flash is illustrated in Figure 2 .

There were no significant differences between infants in the fish oil and placebo groups in b-wave implicit times at any brightness. Moreover, infant cord blood LCPUFA status did not correlate significantly with b-wave implicit time.

The parameters of the Naka-Rushton function, $V_{\max }(\mathrm{F}=$ 2.5, $d f 1,39, P=0.12)$, and $\log \sigma(\mathrm{F}=0.62$, df $1,39, P=0.44)$ did not differ significantly between the fish oil and placebo group (Table 5). The table also shows adult results. In all the infants, regardless of maternal supplementation group, simple linear regression analysis revealed significant correlations between $\log \sigma$ and several measures of DHA status, specifically the proportion (\%TFA) of DHA in RBCs $(r=-0.40$, df 1,36, $P<0.05)$, the total proportion of $\mathrm{n}-3$ fatty acids in RBCs $(r=$ -0.33 , $d f 1,36, P<0.05)$, and the n-6 to n-3 LCPUFA ratio in RBCs $(r=0.36$, df 1,36, $P<0.05)$ of infant cord blood. A similar trend toward negative correlation between $\log \sigma$ and AA (\%TFA) in infant cord blood was observed $(P=0.06)$. Infant LCPUFA status did not correlate significantly with $V_{\text {max }}$.

To establish the robustness of these results and test for nonlinear associations, the Naka-Rushton parameters derived from those infants with lower levels of DHA in their cord blood were compared with those infants who had higher DHA status. The distribution of DHA in RBCs and plasma (\%TFA and concentration) was divided into quartiles (Table 6). A Kendall $\tau$ test was then performed to test for any rank correlation between the Naka-Rushton parameters and RBCs and plasma DHA quartiles. As in the linear regression, higher retinal sensitivity (lower $\log \sigma$ ) was significantly associated with higher DHA levels as measured by quartiles of RBC DHA (\%TFA; $\tau=$ $-0.31, P<0.005$; Fig. 3 ). This nonlinear analysis was repeated for other fatty acids, specifically AA, total n-3 LCPUFAs, total n-6 LCPUFAs, and the n- 6 to n-3 ratio. Similar rank correlations were observed between $\log \sigma$ and the quartile levels of AA $(\tau=$ $-0.24, P<0.05)$ and the sum of n-3 LCPUFAs $(\tau=-0.24, P<$ $0.05)$ in RBCs (\%TFA).

Maximum Combined ERG. The implicit time of the awave $(\mathrm{F}=1.69 ; P=0.2)$ and the $\mathrm{b}$-wave $(\mathrm{F}=0.05 ; P=0.83)$ and the amplitude of the a-wave $(\mathrm{F}=1.1 ; P=0.31)$ and the

TABLE 5. Naka-Rushton Parameters Derived from the Scotopic Intensity Series ERG for Infants in the Fish Oil and Placebo Maternal Supplementation Groups

\begin{tabular}{lcccc}
\hline \multicolumn{1}{c}{ Parameter } & $\begin{array}{c}\text { Fish Oil } \\
(\boldsymbol{n}=\mathbf{2 2})\end{array}$ & $\begin{array}{c}\text { Placebo } \\
(\boldsymbol{n}=\mathbf{1 9})\end{array}$ & $\boldsymbol{P}$ & $\begin{array}{c}\text { Adult Values } \\
(\boldsymbol{n}=\mathbf{1 1})\end{array}$ \\
\hline$V_{\max }(\mu \mathrm{V})$ & $61.9 \pm 30.5$ & $48.6 \pm 21.8$ & 0.12 & $467.6 \pm 113.7$ \\
$\log \sigma(\log$ scot trol s) & $0.39 \pm 0.59$ & $0.26 \pm 0.45$ & 0.44 & $-0.26 \pm 0.22$ \\
\hline
\end{tabular}

Data are the mean $\pm \mathrm{SD}$. 


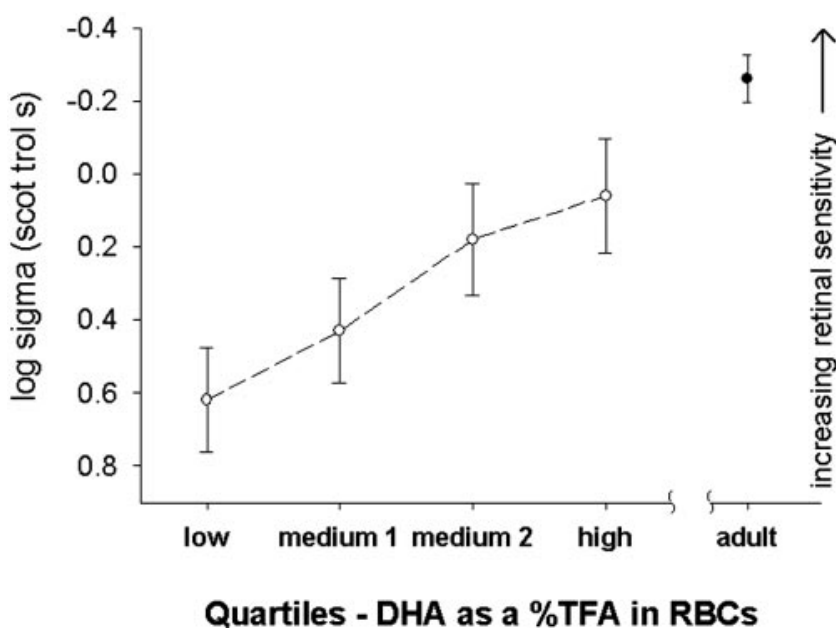

Figure 3. $\log \sigma$ for all infants according to quartiles of DHA in umbilical cord RBCs (\%TFA). The mean \pm SE of $\log \sigma$ in infants in the lowest $(n=9)$, medium $1(n=10)$, medium $2(n=10)$, and highest $(n=9)$ quartiles was $0.62 \pm 0.14,0.43 \pm 0.14,0.18 \pm 0.15$, and $0.06 \pm 0.16$, respectively. $\log \sigma$ values are reversed so that more sensitive retinas are plotted upward. Adult values appear for comparison.

b-wave (F $=0.2 ; P=0.65)$ of the maximal combined ERG did not differ significantly between supplementation groups. Linear regression analysis revealed no significant correlation between implicit times or amplitudes of the a- or b-waves of the maximum combined ERG and infant DHA status at birth. ERGs recorded in infants in the lowest quartile for umbilical cord blood DHA were not distinguishable from the ERGs recorded in those in the highest quartile.

\section{Discussion}

The present study has demonstrated a positive relationship between infant DHA status and the scotopic ERG. Specifically, those infants with the highest DHA levels in RBCs at birth also had the highest retinal sensitivity, regardless of maternal DHA status or supplementation group. However, dietary supplementation of the diets of pregnant women with fish oil rich in DHA from approximately 15 weeks of pregnancy until delivery was associated with no observed enhancement of retinal development of their infants, as measured shortly after birth by scotopic ERG.

The role of dietary LCPUFAs in normal fetal and neonatal visual development has been the focus of an increasing number of clinical studies over recent years. Most of these have examined visual development using electrophysiological methods such as the VEP or behavioral methods such as the Teller acuity card procedure. Few studies have used the ERG to measure visual or, more precisely, retinal function, and all were conducted in preterm infants. ${ }^{11,12,23,24}$ To the authors' knowledge, this is the first published randomized clinical trial to investigating term infant LCPUFA status and retinal development by using the ERG.

In the present study, there were no significant differences in $V_{\max }$ or $\log \sigma$ between infants in the maternal fish oil and placebo supplementation groups. There were, however, a number of significant correlations between infant DHA status and maturity of the scotopic ERGs. Infants with higher retinal sensitivity (based on lower $\log \sigma$ ) had higher DHA in umbilical cord RBCs (\%TFA) than did infants with lower retinal sensitivity. Moreover, the mean retinal sensitivity of infants in the upper quartile for umbilical cord DHA was $0.6 \log$ units higher than that of infants in the lowest quartile for umbilical cord DHA. These findings are in accordance with studies conducted in preterm infants ${ }^{11,12}$ and indicate that increased DHA status in blood is associated with enhanced sensitivity and maturity of the rod photoreceptors.

The lack of a relationship between infant DHA status and the parameter $V_{\max }$ observed in the present study was similar to findings reported by Birch et al. ${ }^{11}$ in their study of preterm infants, in which they found that differences in the maximum b-wave amplitude $\left(V_{\text {max }}\right)$ were marginal in comparison to the differences in $\log \sigma$ between infants fed LCPUFA-enhanced diets and those fed standard formulas. Overall, results agree well with full-field ERG data from slightly older infants. ${ }^{25} \mathrm{How}-$ ever, values obtained in the present study suggest that the newborn retina may be more sensitive and have a larger $V_{\max }$ than predicted by the growth curve analysis of Fulton and Hansen ${ }^{25}$ in which they used data from infants aged 23 days to adults. $^{25}$

The maximum combined ERG was recorded under scotopic conditions in response to a bright white flash and elicited a mixed rod and cone photoreceptor response. No significant differences were observed between supplementation groups in the implicit times or amplitudes of the maximum combined ERG. Moreover, there were no significant correlations between cord blood DHA status and birth and implicit times or amplitudes of the maximum combined ERG. Our findings are again comparable to those of Birch et al. ${ }^{11}$ who reported little difference in the amplitude of ERGs recorded to a maximumintensity white stimulus between infants fed diets differing in LCPUFA content.

In the present study maternal fish oil supplementation during pregnancy had no significant effect on DHA status or retinal development of the neonate measured shortly after birth. It was hypothesized that an increase in the amount of DHA provided in the diet of women during pregnancy would elevate the DHA status of their infants at term. If this were true, increased levels of DHA available to the fetus during the period of rapid brain and retinal growth might contribute to enhanced retinal development. The lack of such a finding suggests that both groups of fetuses obtained the required amounts of LCPUFAs from their mothers to meet the needs of the developing retina. We found that infants in both the maternal supplementation and placebo groups had DHA status and ERG measures within similar normal and acceptable ranges. It is interesting, however, that, even within this healthy cohort of term infants, functional differences in retinal sensitivity were identified between infants with higher levels of DHA in RBC and infants with lower DHA levels. This suggests an association between retinal maturation and DHA accretion by a mechanism that is not based directly on maternal DHA status.

Preterm infants remain at an increased risk of LCPUFA deficiency, because they do not receive the third trimester intrauterine supply of DHA vital for brain and retinal development. In addition, infants born of mothers of low socioeconomic status and mothers with extremely poor diets may also be at increased risk of LCPUFA deficiency and thus altered retinal development. The authors are aware that our study sample consisted of healthy mothers with relatively good dietary habits and that the results may have been different if individuals from more deprived areas could have been included.

Finally, although length of gestation was comparable between supplementation groups, infants in the upper quartiles for DHA in plasma, both as \%TFA and as a concentration, were born at a significantly later gestational ages than were infants in the lower quartiles. Specifically, the length of gestation for infants in the upper quartile for plasma DHA was on average 8 days longer than that of infants in the lowest quartile. Helland 
et al. ${ }^{26}$ reported similar findings in a study of maternal LCPUFA supplementation. In their study, women were supplemented with either cod liver oil or corn oil from weeks 17 to 19 of pregnancy until 3 months after delivery. The results demonstrated longer gestational length, by an average of 7 days, in those infants in the highest quartile for umbilical cord plasma DHA concentration. Such findings are suggestive of a relationship between higher plasma DHA status in the fetus and longer gestation. It should be noted that we found no evidence for an increase in membrane bound DHA in the RBCs, because the length of gestation did not differ when infants were compared based on DHA status in RBCs.

\section{Conclusion}

The present study provides additional evidence that the LCPUFA DHA contributes to the development and function of the visual system and supports the essentiality of this nutrient during early fetal development. Maturation of the retina at birth is enhanced proportionally with the DHA status in the RBCs of umbilical cord blood. Maternal DHA supplementation during pregnancy; however, did not result in any measurable benefits to retinal development in this cohort of healthy term infants.

\section{Acknowledgments}

The authors thank R. P. Scherer, Ltd., for their generous donation of the both the fish oil and placebo capsules and Helen Mactier for expert assistance with the ERG recordings.

\section{References}

1. Fliesler SJ, Anderson RE. Chemistry and metabolism of lipids in the vertebrate retina. Prog Lipid Res. 1983;22:79-131.

2. Stinson AM, Wiegand RD, Anderson RE. Fatty acid and molecular species composition of phospholipids and diacylglycerols. Exp Eye Res. 1991;52:213-218.

3. Clandinin MT, Chappell JE, Leong S, Heim T, Swyer PR, Chance $\mathrm{GW}$. Intrauterine fatty acid accretion rates in human brain: implications for fatty acid requirements. Early Human Development. 1980;4:121-129.

4. Martinez M. Tissue levels of polyunsaturated fatty acids during early human development. J Pediatr. 1992;120:S129-S138.

5. Benolken RM, Anderson RE, Wheeler TG. Membrane fatty acids associated with the electrical response in visual excitation. Science. 1973;182:1253-1254.

6. Wheeler TG, Benolken RM, Anderson RE. Visual membranes: specificity of fatty acid precursors for the electrical response to illumination. Science. 1975;188:1312-1314.

7. Watanabe I, Kato $\mathrm{M}$, Aonuma $\mathrm{H}$, et al. Effect of dietary alphalinolenate/linoleate balance on the lipid composition and electroretinographic responses in rats. Adv Biosci. 1987;62:563-570.

8. Bourre JM, Francios M, Youyou A, et al. The effects of dietarylinolenic acid on the composition of nerve membranes, enzymatic activity, amplitude of electrophysiological parameters, resistance to poisons and performance of learning tasks in rats. $J$ Nutr. 1989;119:1880 - 1892 .

9. Neuringer M, Connor WE, Lin DS, Barstad L, Luck SJ. Biochemical and functional effects of prenatal and postnatal omega-3 fatty acid deficiency on retina and brain in rhesus monkeys. Proc Natl Acad Science USA. 1986;83:285-294.

10. Weisinger HS, Vingrys AJ, Sinclair AJ. The effect of docosahexaenoic acid on the electroretinogram of the guinea pig. Lipids. 1996;31:65-70.

11. Birch DG, Birch EE, Hoffman DR, Uauy RD. Retinal development in very-low-birth-weight infants fed diets differing in omega-3 fatty acids. Invest Ophthalmol Vis Sci. 1992;33:2365-2376.

12. Uauy RD, Birch DG, Birch EE, Tyson JE, Hoffman DR. Effect of dietary omega-3 fatty acids on retinal function of very-low-birthweight neonates. Pediatr Res. 1990;28:485-492.

13. van Houwelingen AC, Sorenson JDJ, Hornstra G, et al. Essential fatty acids in neonates after fish oil supplementation during late pregnancy. Br J Nutr. 1995;74:723-731.

14. Connor WE, Lowensohn R, Hatcher L. Increased docosahexaenoic acid levels in human newborn infants by the administration of sardines and fish oil during pregnancy. Lipids. 1996;31:S183-S187.

15. Montgomery C, Speake BK, Cameron A, Sattar N, Weaver LT. Maternal docosahexaenoic acid supplementation and fetal accretion. BrJ Nut. In press.

16. Malcolm CA, McCulloch DC, Shepherd AJ, Montgomery C, Weaver LT. Effect of maternal docosahexaenoic acid (DHA) supplementation during pregnancy on visual evoked potential development in term infants: a double-blind, prospective, randomised trial. Arch Dis Child. In press.

17. Bradnam MS, Evans AL, Montgomery DMI, et al. A personal computer-based visual evoked potential stimulus and recording system. Doc Ophthalmol. 1994;86:81-93.

18. Birch DG, Fish GE. Rod ERGs in retinitis pigmentosa and cone-rod degeneration. Invest Ophthalmol Vis Sci. 1987;28:140-150.

19. Marmor MF, Zrenner E. Standard for clinical electroretinography (1999 Update). International Society for Clinical Electrophysiology of Vision. Doc Ophthalmol. 1998-1999;97:143-156.

20. Naka KI, Rushton WAH. S-potentials from luminosity units in the retina of fish (Cyprimidae). J Physiol. 1966;185:536-599.

21. Brown AM, Dobson V, Maier J. Visual acuity of human infants at scotopic, mesopic, and photopic luminances. Vision Res. 1987; 27:1845-1858

22. Isenberg SJ, Neumann D, Cheong PYY, Ling YLF, McCall LC, Ziffer AJ. Growth of the internal and external eye in term and preterm infants. Ophthalmology. 1995;102:827-830.

23. Birch EE, Birch DG, Petrig B, Uauy R. Retinal and cortical function of very low birthweight infants at 36 and 57 weeks postconception. Clin Vision Sci. 1990;5:363-373.

24. Leaf A, Gosbell A, McKenzie L, Sinclair A, Favilla I. Long chain polyunsaturated fatty acids and visual function in preterm infants. Early Hum Dev. 1996;45:35-53.

25. Fulton $A B$, Hansen RM. The development of scotopic sensitivity. Invest Ophthalmol Vis Sci. 2000;41:1588-1596.

26. Helland IB, Saugstad OD, Smith L, et al. Similar effects on infants of n-3 and n- 6 fatty acids supplementation to pregnant and lactating women. Pediatrics. 2001;108:E82-E92. 